\title{
Update on the laboratory diagnosis and monitoring of HIV infection
}

\author{
Niel T CONSTANTINE ${ }^{1,3}$, William KABAT ${ }^{4}$, Richard Y ZHAO ${ }^{1-3, *}$ \\ ${ }^{1}$ Department of Pathology, ${ }^{2}$ Department of Microbiology and Immunology, University of Maryland School of Medicine, Baltimore, \\ MD 21201-1192, USA \\ ${ }^{3}$ Institute of Human Virology, Baltimore, MD 21201-1192, USA \\ ${ }^{4}$ Children's Memorial Hospital, Chicago, IL 60614-3363, USA
}

\begin{abstract}
In China, the estimated number of HIV infected cases is approaching one million. Although public education has been initiated for awareness and behavioral modification for this devastating infection, better diagnostic methods are needed to identify infected persons and manage infection. Simple and more accurate diagnostic tools have become available, particularly for early detection and to monitor treatment in those who receive anti-retroviral treatment. In this short review, we summarize some of the common and new methodologies that can be used in clinical laboratories, in the field, or in private laboratories. These range from simple antibody tests to more sophistical methods that are used to monitor disease progression and identify drug resistance. These tools can assist physicians, medical practitioners, and laboratory personnel to select suitable diagnostic tools for the diagnosis, blood screening, monitoring of disease progression, and for detection of drug resistance to anti-retroviral therapies.
\end{abstract}

Keywords: HIV, diagnosis, viral load, drug resistance.

\section{DIAGNOSIS OF HIV-1 INFECTION}

The diagnosis of HIV infection is most commonly accomplished using tests to detect antibody to HIV using a screening test, followed by a supplemental test for confirmation. However, in many countries, including China, screening test reactive samples may not always be confirmed because of the high cost of these assays. In industrialized countries, enzyme immunoassays (EIAs) are the most commonly used methods, while in resourcelimited countries rapid and simple assays are common because of their application in venues where instrumentation or stable electricity are not available. Most EIA methods have excellent sensitivity and good specificity. However, the turn-around time for these standard EIA tests is usually 24 hours, thereby not allowing the return of results with counseling during the patient's initial visit. In China, a popular screening test is a particle agglutination assay (PA). Although this simple-to-perform test does not reduce turnaround times for results, it does allow test-

*Correspondence: Richard Y ZHAO

Tel: 410-706-6301; Fax: 410-706-6302;

E-mail: rzhao@som.umaryland.edu ing without the need for instrumentation and at a low cost.

A number of rapid screening tests are now available that can significantly reduce the testing time to as little as 20 minutes. These rapid HIV tests include dot-blot and immunochromotographic methods. These rapid tests include U.S. FDA approved tests such as the dot-blot based Reveal (MedMira) and Multispot (BioRad) tests, and the lateral flow Uni-Gold Recombigen (Trinity BioTech) and the QraQuick Advance (Orasure Technologies Inc.) tests. Internationally, there are over 100 rapid HIV tests available, many of which can use easily-collected fingerstick whole blood or oral fluids [1]. Oral fluids (saliva) are preferentially collected from the tooth-gum margin and represent a transudate of plasma rather than being pure saliva from the salivary glands [2]. Of particular flexibility is the OraQuick Advance HIV-1/HIV-2 rapid test that can accommodate serum, a drop of fingerstick blood, or oral fluid for testing. This test and the Uni-Gold tests are also approved by the U.S. FDA as CLIA waived tests that allow testing in point of care venues such as physician offices and outreach programs. Besides detecting HIV-1, the OraQuick test allows a claim to detect HIV-2, a viral type that is prevalent in west Africa and has also been found in Chinese patients [3]. 
Although rapid tests are typically more expensive than the standard EIAs, rapid HIV testing offers important advantages that can save lives. In one such case, rapid HIV testing yields results in a clinically-relevant time frame for women in labor who do not have a documented HIV status. Identification of these HIV positive women and therapeutic intervention to the mothers and their infants can substantially reduce vertical transmission from mother to child [4]. Similarly, rapid HIV tests are particularly well suited for bood/body fluid exposures in heath care workers (occupational exposure). Rapid determination of the HIV status of source blood allows for early administration of post-exposure prophylaxis (within 2 hours) with an antiretroviral regimen to the exposed healthcare worker, thereby substantially reducing transmission [5]. Because of the importance of identifying HIV infected persons, several organizations, including the U.S. FDA, are considering to allow rapid HIV tests to be marketed over the counter for home use.

A repeatedly reactive EIA or rapid test result requires confirmation with a suitable supplemental test such as a Western blot, Line Immunoassay (LIA) or immunofluorescence assay (IFA) [1] that are time consuming and expensive, although rapid confirmatory tests have been introduced [6] A positive supplemental test result is evidence of HIV infection, although false positive confirmatory test results have been reported [1]. All supplemental assays may exhibit indeterminate results, usually because of non-specific cross-reacting antibodies, although these may also suggest recent infection that has not yet triggered a vigorous humoral response. In such cases, follow-up testing with serological assays within a few weeks is appropriate and will identify truly infected persons. Alternatively, the clinician might consider re-testing the patient for the presence of HIV by employing a more sensitive nucleic acid-based assay, although these assays are more costly than serological tests.

Unlike in adults, serological testing of children under the age of 18 months has little diagnostic value since maternal antibody can be expected to be present until that time. Nucleic acid-based assays such as the HIV-1 DNA PCR are typically used to screen for HIV infection in children born to HIV infected mothers. Pediatricians will typically screen HIV exposed neonates within the first week of life and again at 1 and 4 months. If a HIV DNA PCR test is positive, the result should be confirmed by a second HIV-1 DNA PCR or a viral load test on a new sample. Children over the age of 18 months that are seropositive for HIV can be confirmed as described for adults.

For assisting with the surveillance of HIV infection, sensitive/less sensitive (S/LS) assays have been introduced to determine the relative time of HIV infection. These assays, based on an assessment of antibody titer or antibody avidity, are modifications of EIAs or rapid tests [7], and can indicate if an infected person were infected within the past 6 months. This allows a calculation of the incidence of infection in a population, thereby allowing the targeting of high risk groups for vaccines or other interventions. S/LS assays have now been developed using a simple particle agglutination assay that is accurate and could be used for field studies in countries such as China (Constantine, unpublished observation).

\section{MONITORING THE EFFICACY OF ANTI-HIV DRUG TREATMENT AND HIV DISEASE PRO- GRESSION}

\section{Viral RNA and DNA}

Treatment of HIV-infected patients with highly active anti-retroviral therapy (HAART) has shown to be very effective in eradicating active replicating viruses from the plasma compartment, and to extend the quality and duration of life $[8,9]$. In addition, clinical data suggest that the viral RNA level at the initial phase of HIV infection (known as the set-point) can be used as a prognostic marker for HIV disease progression [8]. Rapid reduction in viral load $(0.7-3 \operatorname{logs})$ is typically observed in a drug naïve patient within a few weeks after initiation of HARRT. Thus, quantitative measurement of HIV-1 viral load provides a pivotal marker in the disease management of HIV infection, especially in monitoring the efficacy of antiretroviral therapy and disease progression. Quantification of viral RNA levels (viral load) is used by the clinician: 1) to measure the baseline viral load prior to initiation of antiretroviral therapy, 2) to assess the efficacy of initial antiviral drug treatment, 3) to detect the onset of drug resistance both after initial therapy and after prolonged therapy, and 4) to predict the development of AIDS-defining opportunistic infections and disease progression.

There are three major commercial methods that are approved by the U.S. FDA for the measurement of HIV-1 viral RNA load in plasma. They are the Amplicor Monitor ${ }^{\mathrm{TM}}$ by Roche Diagnostics Systems, the Versant ${ }^{\mathrm{TM}}$ HIV RNA kit by Bayer Inc. (formally known as Quantiplex ${ }^{\mathrm{TM}}$ by Chiron Inc.), and the NucliSens ${ }^{\mathrm{TM}}$ HIV-1 QT system by bioMerieux Inc (commonly known as the NASBA assay originally owned by Organon Teknika Co.). The Amplicor Monitor $^{\mathrm{TM}}$ is a RT-PCR based testing system, in which the viral RNA is first reverse transcribed into cDNA and then is amplified through the polymerase chain reaction and detected in DNA form. Similar to the Amplicor Monitor ${ }^{\mathrm{TM}}$ assay, the NucliSens ${ }^{\mathrm{TM}}$ HIV-1 QT assay is a target-based amplification process. However, it directly amplifies the viral RNA using a method mimicking natural replication of retroviruses [10]; Unlike these target-based assays, the 
Versant ${ }^{\mathrm{TM}}$ HIV RNA method is based on the amplification of the signal generated after detection (i.e., a signal-amplification based assay). This is accomplished by first capturing the viral RNA targets, followed by sequential oligonucleotide hybridizations [11]. In this assay, a specially designed branched-DNA (bDNA) probe is used in conjunction with the primary capturing probe to further enhance the signal amplification; hence, it is also known as the bDNA assay.

All three commercial methods achieve similar results in the quantification of HIV-1 viral RNA load [12]. The lower detection limit is 400-500 RNA copies per mL of plasma, with a detection range from 3 to 5 logs. All three assays also have an ultra-sensitive version which extends the lower detection limit to about 50 copies of viral RNA copies per $\mathrm{mL}$ of plasma. However, usage of these ultra-sensitive assays are somewhat limited by the requirement for larger plasma volumes $(0.5-2 \mathrm{~mL})$, thereby placing a limitation for pediatric viral load determinations. By contrast, standard assays typically require $100-200 \mu \mathrm{L}$ of plasma. A smaller $50 \mu \mathrm{L}$ format of the bDNA assay is also available which is particularly useful in pediatric testing [13].

In addition to these three popular assays, a number of other methods, such as ligase chain reaction (LCR, Abbott Laboratories), hybrid capture system (Digene Co.), transcription mediated amplification (TMA, Gen-Probe Inc.; approved by US FDA for qualitative testing and blood screening), and a reverse transcriptase viral load method (ExaVir Load, Cavidi Tech, Uppsala Sweden) are also commercially available. A SYBR green-based real-time RT-PCR method using the LightCycler technology was also reported recently [14]. Although each is based on a different scientific principle, they all provide similar results, although it is recommended not to use the results from more than one assay for managing each patient.

In addition to nucleic acid-based assays that can be used to monitor HIV-1 viral load and disease progression, there are several methods that have been used to monitor patients. These include various serologic HIV-1 p24 antigen detection methods (including an amplification-boosted ELISA) [15], qualitative and quantitative cell culture methods, and enumeration of T-helper (CD4) and T-suppressor (CD8) cells by flow cytometry. Currently, quantitative viral load and CD4/CD8 determinations represent the most widely used laboratory assays employed in HIV patient management. Recently, a report describes a real-time immuno-PCR (IPCR) assay that uses HIV-1 p24 antigen as a marker for quantification of viremia [16]. The realtime IPCR assay combines the p24 ELISA with real-time PCR amplification of the signal DNA molecules that are attached to the detecting antibody of p24. The testing of patient samples, which had an undetectable viral RNA load (i.e., $<50$ viral RNA copies/ml), showed that IPCR detected viremia in $42.3 \%$ of these patients with detectability at the femtogram $/ \mathrm{mL}$ level. Thus, fine tuning of this technology could potentially result in one of the most analytically sensitive tests for the detection of HIV-1 viral load through the detection of HIV-1 p24 antigen.

Even though patients starting HAART regimens often achieve non-detectable HIV-1 viral RNA levels, questions remain as to whether or not the proviral DNA, either integrated or un-integrated, responds to HAART in infected patients. Do relative changes in proviral DNA levels precede changes of viral RNA levels post HAART induction? Therefore, one of the current challenges in the HIV disease management is to detect the presence of low level proviral DNA in latently-infected CD4+ lymphocytes and other reservoirs, especially because these hidden reservoirs can replenish and revive viral infection upon activation [17-19]. Thus, a highly reproducible and accurate assay to quantify proviral DNA would enable a more in-depth evaluation of the efficacy of antiviral therapies. Unfortunately, there are no commercially available assays currently available for quantification of HIV-1 proviral DNA levels. Several research-based assays have been reported previously for quantification of HIV-1 proviral DNA, all of which were based on the principle of conventional PCR [20-23]. The potential limitation associated with the traditional quantitative PCR is that the DNA copy numbers are calculated based on the final amplified gene products. Since DNA is amplified exponentially during PCR, a small variation in amplification efficiency early in the thermocycling process could potentially lead to a large variation in the final quantification of amplified products. To circumvent this problem, a TaqMan-based protocol for quantification of HIV-1 proviral DNA was reported [24]. This assay is based on the principle of real-time PCR, which is also known as $\operatorname{TaqMan}^{\mathrm{TM}}$ [25]. Due to the inherent advantage of real-time PCR over classic PCR, this new assay provides a highly accurate and reproducible detection method for HIV-1 proviral DNA with a broad linear range of detection [24].

\section{CD4 levels}

A number of methods are available to measure CD4 levels to monitor the immune system during the course of HIV infection. Although flow cytometry is considered the reference method, it requires significant technical expertise, expensive instrumentation, and is costly. Alternatives have become available that are simpler to perform, portable, and can be performed at a much reduced cost. Some require only a light microscope and cell counting, while others are based on EIA technology. These include: Cytospheres (Coulter), Dynabeads (Dynal Biosciences), 
Opti-CIM (CIMA), Zymmune (Zynaxis), TRAxCD4 (T Cell Dxs, and Innogenetics), CD4 Count Chip (SemBio), the Capcellia (Sanofi), and the CD4 Biochip (LabNow). Most methods are inexpensive and cost between \$3-10 per test, as compared with $\$ 25-40$ per test for flow cytometry. Information on these methods has been published [1].

\section{DETECTION OF HIV-1 DRUG RESISTANCE AND MONITORING OF TREATMENT FAIL- URES}

The Chinese government implemented a free antiretroviral therapy (ART) program in 2003. Under this new policy, a selected group of Chinese HIV-infected patients are being treated with ART. Due to limited number of antiviral regimens, current ART is limited to the combined use of few drugs including AZT, ddI, d4T, 3TC, NVP, IDV and EFV. Nevertheless, drug resistance will start to emerge and is soon going to be a major problem as in the rest of the world. Thus, it is crucial to detect drug resistant viruses early and switch ART when alternative regimens are available or necessary. In spite of the striking success of ART in treating HIV-infected patients, most patients will eventually fail treatment, as genetic changes emerge in the virus leading to drug resistance. The observed resistance is usually restricted to changes in those viral genes that serve as targets for the therapies that the patient has experienced. For example, patients taking AZT (zidovudine) as part of their therapy will typically develop mutations of codon 215 of the reverse transcriptase region of the HIV pol gene leading to a change in a single amino acid of the gene product and decreasing effectiveness of AZT in the patient. Over time it is likely that additional mutations will occur (while the patient is taking AZT) in codons such as 41 and 219, again, leading to different amino acid changes in the reverse transcriptase protein. The cumulative acquisition of new mutations is likely to lead to a loss of effectiveness of AZT [26, 27] that can be demonstrated by HIV-1 genotyping, high inhibitory levels in phenotypic drug susceptibility assays, and increasing levels of HIV viral load.

For the clinician, the first suggestion indicating drug failure is a longitudinal trend of increasing viral loads over a short period of time. When this occurs, the clinician must first establish that the patient is taking his/her medications in the appropriate doses and at the scheduled times. Some HIV treatment regimens are very difficult for patients or care givers to manage and many documented drug failures have shown to be directly related to noncompliance. There are a number of other ways to determine emergence of drug resistance in patients undergoing antiviral therapy. Genotypic analysis of the patient's cir- culating viruses in plasma is the most direct method to determine if viral gene changes are responsible for the loss of antiretroviral therapy. Since regimens currently used in HAART are combinations of HIV-1 RT and PR inhibitors, gene mutations in the RT and PR genes are most likely responsible for HIV drug resistance. The use of automated DNA cycle-sequencing based-technology is currently the most common way to conduct HIV-1 genotyping analysis. Both the ViroSeq ${ }^{\mathrm{TM}}$ HIV-1 Genotyping Systems (PE Applied Biosystems) and the Trugene ${ }^{\mathrm{TM}}$ HIV-1 test (Visible Genetics) offer such tests with an integrated software algorithm to provide information to the physician about which gene mutations of RT or PR are most likely to confer resistance to a specific drug regimen. Inter-laboratory comparison of various DNA sequencing-based genotypic methods indicated that there is a high concordance in determining RT and PR gene mutations [28]. Results of a recent study designed to compare the ability of different technologies in detecting minor variants of HIV RT or PR mutations indicated that a number of other techniques may have at least similar if not better capacity in detecting HIV gene mutations. These techniques include allele-specific real-time PCR (ASRT), a Ty1/HIV-1 RT yeast hybrid assay (Ty1), Oligonucleotide ligase-based assay (OLA), limiting dilution RT-PCR (LD RTPCR), and Pyrosequencing [29]. Several less costly assays with limited testing capability are also available to provide similar information on HIV-1 drug resistance. For example, the LiPA ${ }^{\mathrm{TM}}$ assay (Bayer Co. formally by Innogenetics) uses easy read-out strips for determination of specific viral gene codon changes conferring drug resistance, but the use of this test only limits interpretation to the gene mutations printed on the strip. Similarly, the use of the DNA repair enzyme Cleavase $^{\mathrm{TM}}$ in the Invader ${ }^{\mathrm{TM}}$ assay (Third Wave Technology) could potentially detect all of the viral gene mutations, but it is unable to provide specific information on gene codon changes. One of the most powerful genotypic ways to determine HIV-1 drug resistance is the use of microarray technology. Affimatrix has developed a specific GeneChip HIV PRT Probe Array ${ }^{\mathrm{TM}}$ that can be used to detect all of the possible gene mutations conferring drug resistance to either RT or PR inhibitors. However, this assay is limited by its high cost in using the chips and instrument setup.

Results of the genotypic analysis are compiled for the physician but it is up to the physician to decide if therapeutic changes are needed for the patient. This decision is often made based on the physician's personal experience and the evolving treatment literature. Drug resistant profiles of HIV-1 to all of the current anti-HIV regimens have been updated annually by the International AIDS SocietyUSA Drug Resistance Mutation Group (For its 2003 recommendations, see [30]). Some clinicians are inclined 
Tab. 1 Summary of HIV-1 Diagnostic Methods

\begin{tabular}{|c|c|c|c|c|}
\hline \multicolumn{5}{|c|}{ I. Diagnosis of HIV-1 Infections } \\
\hline Target of detection & Detection methods & Commercial tests & Manufacture & U.S. FDA-approved \\
\hline \multirow[t]{6}{*}{ Anti-HIV Antibody } & EIA & EIAs & Abbott/Coulter & $\mathrm{Y}$ \\
\hline & Rapid EIA & SUDS & Abbott/Murex & $\mathrm{Y}$ \\
\hline & Rapid EIA & OraQuick & OraSure & $\mathrm{Y}$ \\
\hline & Rapid EIA & Reveal & MedMira & Y \\
\hline & Rapid EIA & Uni-Gold Recombigne & Trinity BioTech & $\mathrm{Y}$ \\
\hline & Western blotting & Cambridge Biotech WB & Calypte Biomedical & $\mathrm{Y}$ \\
\hline Viral DNA & DNA PCR & Amplicor & Roche & $\mathrm{N}$ \\
\hline Viral RNA & RNA PCR & see next section & & \\
\hline \multicolumn{5}{|c|}{ II. Monitoring HIV Disease Progression } \\
\hline Target of detection & Detection methods & Commercial tests & Manufacture & U.S. FDA-approved \\
\hline \multirow[t]{9}{*}{ Viral RNA } & RNA PCR & Amplicor Monitor & Roche & $\mathrm{Y}$ \\
\hline & b-DNA & VERSANT & Bayar & $\mathrm{Y}$ \\
\hline & NASBA & NucliSen HIV-1 QT & bioMerieux & $\mathrm{Y}$ \\
\hline & TMA & Procleix & Gen-Probe & $\mathrm{Y}^{*}$ \\
\hline & LCR & $\mathrm{LCX}$ & Abbott & $\mathrm{N}$ \\
\hline & SDA & SDA & Becton Dickinson & $\mathrm{N}$ \\
\hline & Qb-replicase & QbR & Gene-Track & $\mathrm{N}$ \\
\hline & Hybridization & Hybrid Capture System & Digene & $\mathrm{N}$ \\
\hline & RT & ExaVir Load & Cavidi Tech & $\mathrm{N}$ \\
\hline Viral DNA & Real-time PCR & $\mathrm{n} / \mathrm{a}$ & $\mathrm{n} / \mathrm{a}$ & $\mathrm{n} / \mathrm{a}$ \\
\hline \multicolumn{5}{|c|}{ III. Detection of HIV-1 Drug Resistance } \\
\hline Target of detection & Detection methods & Commercial tests & Manufacture & U.S. FDA-approved \\
\hline \multirow{6}{*}{$\begin{array}{l}\text { RT and Protease } \\
\text { (Genotyping) }\end{array}$} & DNA sequencing & ViroSeq & PE Applied Bios. & $\mathrm{Y}$ \\
\hline & DNA sequencing & Trugene & Visible Genetics & $\mathrm{Y}$ \\
\hline & Reverse hybridization & LiPA & Innogenetics & $\mathrm{N}$ \\
\hline & DNA repair by cleavase & Invader & Third Waves & $\mathrm{N}$ \\
\hline & Microarray & GeneChip & Affymetrix & $\mathrm{N}$ \\
\hline & In vitro drug sensitivity & PhenoSense & ViroLogic & $\mathrm{N}$ \\
\hline
\end{tabular}

*, The Procleix test is approved by FDA as a qualitative test only; Y, yes; N, no; n/a, not applicable

to use phenotypic HIV assays as they directly measure the viral drug susceptibility. Furthermore, phenotypic assays are also able to determine multidrug cross-resistance. Currently, ViroLogic in the U.S. and Virco in Belgium provide this service. The Phenosense ${ }^{\mathrm{TM}}$ assay measures antiviral drug susceptibility in a cell culture based assay using viral constructs that have been derived by splicing patient viral pol gene sequences into a well-characterized viral vector. The new virus with patient viral genes is used to infect a matched cell line in the presence of several different antiretroviral drugs across therapeutically important ranges. Replication of the patient's virus is compared to a wild type sensitive viral control and the virus is scored as "sensitive" or "resistant". In addition, ViroLogic also offers a new Replication Capacity (RC) assay (also known as the Viral Fitness assay), which measures the ability of a an individucal's HIV to make copies of itself and is designed to provide useful additional information to physicians to select optimal antiretroviral therapy cocktails for their patients. A drawback of the Viral Fitness and Phenotypic HIV assays is that they are currently much more expensive than the genotyping assays and have a turn- 
around-time of 2-4 weeks. To circumvent this problem, some companies now offer genotypic testing with a "virtual phenotype". The virtual phenotype is generated after the genotype is completed by comparing a large database of genotypic results with correlated phenotypic test outcomes.

\section{SUMMARY}

Methods for the laboratory diagnosis and monitoring of HIV infection have evolved and offer a large number and variety of effective methods that can extend the quality of life for HIV infected persons (Tab. 1). However, many of these new methods are expensive and require technical expertise, but alternatives have become available that are suitable for many different testing venues. In China, many of these technologies are being considered and may even be used in select laboratories. However, they are not routinely used for patient management. Because of the estimated large number of HIV infected Chinese persons, and the likelihood for an explosive increase in numbers over the next decade, it is critical for these methods to be considered for adoption, particularly for monitoring the response to therapy and identifying emerging viral resistance to drugs.

\section{REFERENCES}

1 Constantine NT, ed. Retroviral Testing and Quality Assurance: Essentials for Laboratory Diagnosis. Ann Arbor, MI: Malloy Publishers, 2005.

2 Branson BM. FDA approves OraQuick for use in saliva. On March 25, the FDA approved the first rapid test for HIV in oral fluids. AIDS Clin Care 2004; 16:39.

3 Update CHATPN. Uncommon HIV-1 Virus Detected in China. Kyodo News Services. 2000.

4 Bulterys M, Jamieson DJ, O'Sullivan MJ, et al. Rapid HIV-1 testing during labor: a multicenter study. JAMA 2004; 292:21923.

5 Prevention CfDCa. CDC: better needlestick reporting required. AIDS Alert 1997; 30-1.

6 Constantine NT, Ketema F. Rapid confirmation of HIV infection. Int J Infect Dis 2002; 6:170-7.

7 Constantine NT, Sill AM, Jack N, et al. Improved classification of recent HIV-1 infection by employing a two-stage sensitive/ less-sensitive test strategy. J AIDS 2003; 32:94-103.

8 Albrecht H, Hoffmann C, Degen O, et al. Highly active antiretroviral therapy significantly improves the prognosis of patients with HIV-associated progressive multifocal leukoencephalopathy. AIDS 1998; 12:1149-54.

9 de Mendoza C, Soriano V, Perez-Olmeda M, et al. Different outcomes in patients achieving complete or partial viral load suppression on antiretroviral therapy. J Hum Virol 1999; 2:344-9.

10 Guatelli JC, Whitfield KM, Kwoh DY, Barringer KJ, Richman $\mathrm{DD}$, Gingeras TR. Isothermal, in vitro amplification of nucleic acids by a multienzyme reaction modeled after retroviral replication. Proc Natl Acad Sci U S A. 1990; 87:1874-8.
11 Urdea MS, Horn T, Fultz TJ, et al. Branched DNA amplification multimers for the sensitive, direct detection of human hepatitis viruses. Nucleic Acids Symp Ser 1991; 197-200.

12 Shingadia D, Zhao Y. Mesurement of plasma viral RNA load of human immunodeficiency virus type 1 (HIV-1). Am Med Lab Int 1997; 2:4-5.

13 Yeghiazarian T, Zhao Y, Read SE, et al. Quantification of human immunodeficiency virus type 1 RNA levels in plasma by using small-volume-format branched-DNA assays. J Clin Microbiol 1998; 36:2096-8.

14 Gibellini D, Vitone F, Gori E, La Placa M, Re MC. Quantitative detection of human immunodeficiency virus type 1 (HIV-1) viral load by SYBR green real-time RT-PCR technique in HIV-1 seropositive patients. J Virol Meth 2004; 115:182-9.

15 Schupbach J, Boni J, Flepp M, et al. Antiretroviral treatment monitoring with an improved HIV-1 p24 antigen test: an inexpensive alternative to tests for viral RNA. J Med Virol 2001; 65: 225-32.

16 Barletta JM, Edelman DC, Constantine NT. Lowering the detection limits of HIV-1 viral load using real-time immuno-PCR for HIV-1 p24 antigen. Am J Clin Pathol 2004; 122:20-7.

17 Chun TW, Stuyver L, Mizell SB, et al. Presence of an inducible HIV-1 latent reservoir during highly active antiretroviral therapy. Proc Natl Acad Sci U S A 1997; 94:13193-7.

18 Finzi D, Blankson J, Siliciano JD, et al. Latent infection of CD4+ $\mathrm{T}$ cells provides a mechanism for lifelong persistence of HIV-1, even in patients on effective combination therapy. Nat Med 1999; 5:512-7.

19 Finzi D, Hermankova M, Pierson T, et al. Identification of a reservoir for HIV-1 in patients on highly active antiretroviral therapy. Science 1997; 278:1295-300.

20 Bennett JM, Kaye S, Berry N, Tedder RS. A quantitative PCR method for the assay of HIV-1 provirus load in peripheral blood mononuclear cells. J Virol Methods 1999; 83:11-20.

21 Christopherson C, Kidane Y, Conway B, Krowka J, Sheppard $\mathrm{H}$, Kwok S. PCR-Based assay to quantify human immunodeficiency virus type 1 DNA in peripheral blood mononuclear cells. J Clin Microbiol 2000; 38:630-4.

22 Guenthner PC, Hart CE. Quantitative, competitive PCR assay for HIV-1 using a microplate-based detection system. Biotechniques 1998; 24:810-6.

23 Izopet J, Tamalet C, Pasquier C, et al. Quantification of HIV-1 proviral DNA by a standardized colorimetric PCR-based assay. J Med Virol 1998; 54:54-9.

24 Zhao Y, Yu M, Miller JW, et al. Quantification of human immunodeficiency virus type 1 proviral DNA by using TaqMan technology. J Clin Microbiol 2002; 40:675-8.

25 Holland P, Abramso R, Watson R, Gelfand D. Detection of specific polymerase chain reaction product by utilizating the $5^{\prime}-3^{\prime}$ exonuclease activity of Thermus aquaticus DNA polymerase. Proc Natl Acad Sci U S A 1991; 88:7276-80.

26 Larder BA, Darby G, Richman DD. HIV with reduced sensitivity to zidovudine (AZT) isolated during prolonged therapy. Science 1989; 243:1731-4.

27 Larder BA, Kemp SD. Multiple mutations in HIV-1 reverse transcriptase confer high-level resistance to zidovudine (AZT). Science 1989; 246:1155-8.

28 DeMeter LM, D'Aquila R, Weislow O, et al. Interlaboratory concordance of DNA sequence analysis to detect reverse tran- 
scriptase mutations in HIV-1 proviral DNA. ACTG Sequencing Working Group. AIDS Clinical Trials Group. J Virol Methods 1998; 75:93-104.

29 Halvas E, Advovand G, Balfe I, et al. Blinded, multicenter comparison of the sensitivity of different technologies to detect and quantify a minor drug-resistant HIV-1 variant. The. Paper presented at: 10th Conference on Retrovirus and Opportunistic
Infections, 2003; Boston.

30 Hirsch MS, Brun-Vezinet F, Clotet B, Richman DD. Antiretroviral Drug Resistance Testing in Adults Infected with Human Immunodeficiency Virus Type 1: 2003 Recommendations of an International AIDS Society-USA Panel. Clin Inf Dise 2003: 113-28. 